\title{
Manifestation einer alternativen Rechtskultur: 2. Alternativer Juristentag
}

Schon seit etwa 2 Jahrzehnten sehen gesellschaftlich und politisch engagierte Juristen im Deutschen Juristentag (DJT) kein Forum für gesellschaftsbezogene Rechtsreformen mehr'. Diese Juristen zu einer gemeinsamen Tagung zu versammeln, wie eben beim DJT, erwies sich als sehr schwierig. So waren selbst die Protagonisten überrascht, im vergangenen Jahr beim I. Alternativen-Juristentag (AJT) fast 250 Teilnehmer zu finden², eine Zahl, die vom 29. November bis I. Dezember I99 I trotz des geringen zeitlichen Abstands - in Zukunft ist ein zweijähriger Turnus, im Jahr zwischen den DJ-Tagen, vorgesehen - auf fast 400 angestiegen war, darunter der niedersächsische Ministerpräsident Gerhard Schröder. Er nahm auch das Wort: Keine der üblichen Begrüßungsansprachen, sondern ein persönliches Wort zu den Problemen, die heute einen engagierten Juristen bewegen.

Ein Gegenjuristentag also, der sich mit dem schicken »Alternativ«-Mäntelchen schmückt? Kaum. Nirgendwo ist wohl im Rechtsbereich "Alternativ « so zutreffend wie hier. Die durchaus ernstzunehmende Befürchtung aus einem Kreis progressiver Richter, auch hier würden »RichterInnen ... zum Kopfsteinpflaster für die Auftritte von Festspielhelden ${ }^{3}$ - also repräsentativer »linker « Rechtslehrer - wurde nicht wahr. Die Diskussion wurde von der »Basis«, von nicht bekannten Juristen, ja Noch-nicht-Juristen, Studenten und Referendaren, bestimmt und auch nicht von Wissenschaftlern, sondern von Praktikern aus Gerichten, Behörden, auch Betrieben und nicht zuletzt aus der Anwaltschaft. Juristinnen waren nicht nur Dekoration, beim DJT eigentlich dem Damenprogramm zugedacht, sondern stellten nahezu die Hälfte der Teilnehmer, waren also im Vergleich zu ihrem Anteil an der Juristenschaft überrepräsentiert. Alternativ ist auch die organisatorische Gestaltung. Der AJT hat keinen juristischen Mantel, hat nicht einmal trotz der in der Einladung genannten unterstützenden Personen und Organisationen einen formellen Veranstalter. Er läuft gewissermaßen spontan $a b .{ }^{4}$

Der AJT bewirkt nicht nur die Festigung des Selbstverständnisses aufgeschlossener Juristen und Handreichung für den Berufsalltag, in dem sie sich als Minderheit unter unpolitischen und damit unspezifisch konservativen Juristen befinden, sondern ist vor allem eine Manifestation einer alternativen Rechtskultur. In einer Zeit der Defensive der progressiven Intelligenz, ihrer Zersplitterung in Gruppen und Einzelpersonen, ist es ausgerechnet im konservativ geprägten Rechtsbereich zu einer Offensive gekommen, bei einer eher individualistisch ausgerichteten Profession zu einer Sammlung von auf Gruppen und Grüppchen verteilten Juristinnen und Juristen, von der SPD und den Grünen, über den Richterratschlag, der ÖTV in der Rechtspflege, der Neuen Richtervereinigung, dem Republikanischen Awältinnen- und Anwälteverein, bis zur Humanistischen Union, um die wichtigsten Gruppen zu nennen, zu denen viele institutionell ungebundene hinzukommen. Eine Rechtskultur, die sich

I Vgl. Theo Rasehorn, Empfiehlt es sich, einen Alternativen Juristentag einzurıchten?, KJ I99I, $69 \mathrm{ff}$

2 Vgl. die vom Republikanischen Anwaltunnen- und Anwalteverein, Hannover, herausgegebene Dokumentation Alternativer Juristentag 23.-25. November 1990.

3 Klaus Beer, Skepsis gegenuber dem "Alternativen Juristentag“, Betrifft Justiz 1989, 98.

4 Wenn gleichwohl die Organisation reibungslos war, so ist dies vor allem ein Verdienst von Rechtsanwal Bertram Borner und Rechtsan walltin Margarete Fabricius-Brand, ohne deren personliches Engagement es wohl nicht zum AJT gekommen ware. 
u. a. in der Diskussion und damit auch als Streitkultur zeigt, aber zugleich in der Kommunikation, in einem mitmenschlichen Miteinanderumgehen - weitab von dem distanziert bürokratischen Gehabe bei den meisten juristischen Institutionen -, ein gewichtiger Grund wohl für die Anziehungskraft des AJT bei Juristinnen.

\section{Keine rechtswissenschaftliche Tagung}

Weil bewußt keine »Papiere« produziert, keine Beschlüsse gefaßt wurden - bis auf eine Resolution gegen den Entwurf des Justizentlastungsgesetzes -, ist es schwer, den AJT in die "klassische " rechtswissenschaftliche Diskussion einzufügen.' Dies mag weiter durch die geringe Teilnahme progressiver Rechtswissenschaftler erschwert sein. Diese waren es doch gerade, die nach 1968 lange Jahre das "linke Lager « anführten. Seit einem Jahrzehnt haben sie sich aber wohl auf den universitären Bereich zurückgezogen, agieren hier als Gesprächspartner und Widerpart von unpolitisch konservativen Wissenschaftlern.

Allerdings kamen viele Referenten aus dem wissenschaftlichen Bereich. Ihre Themen setzten an der Schnittstelle von Recht, Gesellschaft und Politik an. Ein Grund mehr, den Juristentag als einen alternativen zu bewerten, ein Grund aber auch, daß sich daraus nur schwer neue rechtswissenschaftliche Erkenntnisse abscheiden lassen.

\section{Themen und Diskussion}

Der inhaltliche Tagungsbericht darf unter Hinweis auf die geplante Dokumentation knapp erfolgen. Zum Thema "Gleichstellung von Frauen «, angekündigt als Beschreibung der »Falle für Frauen « wegen der Zuständigkeit für »Kinder, Hausarbeit und Gefühle «, um dem »Mann den Rücken freizuhalten «, referierte mit Frigga Haug zunächst eine Soziologin. Oben stünden die Männer als »Eingeweihte«, unten Frauen und Kinder als Volk. Aber gehört nicht der »einfache « Mann auch zum Volk, ist er heute als >Pseudoeingeweihter « nicht eher überfordert? Juristischer wurde es bei Ute Gerhard, die im Hinblick auf die Bedeutung des Rechts eher eine Gegenposition vertrat, das Recht nicht als Machtpotential der Eingeweihten, sondern als Chance zum Nutzen des Volkes auffaßte.

Beim Podium für die Ausländerdiskussion, angekündigt: "Mitmenschen in Not, abweisen oder helfen? « war nur der Diskussionsleiter, Eckart Hien, Richter am Bundesverwaltungsgericht, Jurist. Es referierten zwei Politiker: Antje Vollmer und Reinhart Klimmt, SPD-Fraktionsvorsitzender im Saarland. Hinzu trat Rosi WolfAlmanasreh, Leiterin des Amtes für multikurelle Angelegenheiten, Frankfurt a. M. Es überrascht nicht, daß die Referenten, so sehr auch die Themenbehandlung unterschiedlich war, in den Ergebnissen bei der Asyl- und Einwanderungsfrage im wesentlichen übereinstimmten. Diese Selbstvergewisserung mußte im Hinblick auf die Exzesse gegen Ausländer und die latente Ausländerfeindlichkeit auch sonst im gesellschaftlich-politischen Raum ermutigend empfunden werden.

Eher resigniert verlief dagegen die Diskussion zum Umweltproblem. Noch mit einem Hauch Optimismus Georgios Magoulas, der die Meisterung der Schadstoffproblematik durch die Wirtschaft - und zu derem Nutzen - als aussichtsreich bezeichnete. Ulrike Riedel, die als Staatssekretärin ihren verhinderten Umweltminister Joschka Fischer vertrat, informierte über die Auseinandersetzung mit der Atomindustrie in Hessen. Skepsis vor allem bei Petra Kelly in ihrem Referat über ökologi- 
sche Kinderrechte. Beim rechtlichen Part sah Rainer Wolf das Umweltrecht in einer Krise.

Als Höhepunkt, auch nach der Bewertung in den Medien, erwies sich das Thema »Unrecht, Versagen und Schuld in der DDR «. Dabei ist aber leider die Kontroverse zwischen dem politisch-juristischen Referat der Berliner Justizsenatorin Jutta Limbach und dem anthropologischen des Pfarrers Friedrich Schorlemmer nicht ausgetragen worden. Jene referierte über das Erbe einer totalitären Parteidiktatur im Lichte des Rechtsstaates. Dabei mußte sich die Radbruch-Formel zum NS-Unrecht aufdrängen: Der Widerspruch des positiven Gesetzes zur Gerechtigkeit könne ein so unerträgliches $\mathrm{Maß}$ erreichen, daß das Gesetz als »unrichtiges Recht « der Gerechtigkeit zu weichen habe. ${ }^{6}$ So müsse sich auch hier der Rechtsstaat aufgeben, wenn er die Verfolgung der »Mauerschützen« erst von dem Ausgang von Strafverfahren gegen deren Befehlsgeber, vor allem also Erich Honecker, abhängig mache. Schorlemmer setzte die rechtliche Bewältigung hinten an; ihm ging es vor allem um die »Versöhnung in der Wahrheit «.? So war es schwer, eine Diskussionsbrücke zwischen den Referaten zu finden. Zudem mußte Schorlemmer in seiner Person und essayistischen Darstellung, als Redner ohne die Routine »westlicher « Referenten, unverbraucht, merkbar befreit, in der Öffentlichkeit ungehindert reden zu dürfen, zum Mittelpunkt der Diskussion werden. Bei dem anschließenden nur von der Ex-DDR besetzten Podium zeigte sich dagegen einmal mehr die Unsicherheit der dortigen Menschen, die Furcht gleicherweise vor der Vergangenheit wie vor der Zukunft. Jeder, auch Schorlemmer, schloß das Auftauchen von Dokumenten nicht aus, die, vom Westen aus gesehen, zur eigenen Belastung führen könnten. Die Liedersängerin Barbara Thalheim wies darauf hin, die Staatssicherheit sei ja zugleich eine Behörde gewesen, mit der bei Westreisen Kontakt aufzunehmen gewesen sei. Wer mag da ausschließen, daß ein Stasi-Offizier, um bei seinen Vorgesetzten gut anzukommen, Informationen aufgebauscht hat? Vorsicht bei der Auswertung der Akten ist geboten, wie sich ja auch aus dem Fall des Universitätsrektors Fink ergibt.

Wie sich Westdeutsche mit den Problemen Ostdeutscher bei der Bewältigung des DDR-Unrechts schwer tun, so mag diesen die Sensibilität für die Nachwirkung der NS-Justiz im Westen fehlen. So verstieg sich ein Rechtsanwalt und SPD-MdL des brandenburgischen Landtags zu der These, gerade die Wiedereinstellung der NS(Mord)Richter habe den Rechtsstaat in der Bundesrepublik gefördert. Verkannt wird, daß hier nur eine Wunde zugedeckt worden ist, die sich wohl erst nach einem Jahrhundert schließen kann, wenn die NS-Justiz zur Historie geworden ist.

\section{Wie soll es weiter gehen?}

Gewiß hat sich bei den Alternativen Juristentagen eine alternative Rechtskultur manifestiert; aber Beständigkeit ist noch nicht gewährleistet. Es gibt noch keine Brücke zu den Juristen in den neuen Ländern. Trotz des DDR-Themas und der Nähe des Tagungsortes für Ostdeutsche zählten zu diesen nur 10\% der Teilnehmer. Dabei mögen die Hälfte noch abgeordnete Westdeutsche gewesen sein. Rechtswissenschaftler - deren Dominanz manche im Vorfeld zu Unrecht befürchtet hatten ${ }^{8}-$ und Sozialwissenschaftler sollten nicht nur als Referenten, sondern auch als allgemeine

6 Das Bundesverfassungsgericht war aber repräsentativ vertreten.

7 Gustav Radbruch: Gesetzliches Unrecht und ubergesetzliches Recht, SJZ 1946, 105.

8 Das Referat ist fast vollstandıg in der FAZ 2. 1 2. 199 I wiedergegeben. Dabei war auch der Anlaß genannt, allerdings laßt die eigene Bezeichnung: «alternativer " Juristentag die zu erwartende Distanz erkennen. 
Diskussionsteilnehmer stärkere Impulse setzen. Es müßten auch mehr am Rechtswesen interessierte Laien gewonnen werden. ${ }^{9}$

Der Rahmen der ersten beiden Juristentage reicht nicht mehr. Im Hinblick auf unsere tagungsübersättigte Zeit war jedes Mal mit weit geringeren Teilnehmerzahlen gerechnet worden, weshalb die Allgegenwart von Plenumsdiskussionen geboten wurde. Bei einem Plenum von über 300 Personen ist dies aber aus objektiven und subjektiven Gründen - nicht jeder und vor allem nicht jede kann da unbefangen sprechen - schwer möglich. Da nach den Erfahrungen beim nächsten AJT über 400 Teilnehmer einkalkuliert werden müssen, sollten neue Formen der Diskussion und Kommunikation geplant werden, ohne daß es zu der sonst üblichen Zersplitterung in Gruppen mit ihrem Eigenleben kommt. Ein Alternativer Juristentag sollte sich nicht mit Tradition und Routine zufrieden geben, sondern für Experimente und Innovation offen sein.

9 Vgl. Beer (Fn. 3)

Io So Rasehorn (Fn. I), S. 75 . 